\title{
THE SCHUR SUBGROUP OF THE BRAUER GROUP OF CYCLOTOMIC RINGS OF INTEGERS
}

\author{
C. RIEHM
}

(Communicated by Bhama Srinivasan)

\begin{abstract}
Let $K$ be a finite abelian extension of the rational numbers $\mathbb{Q}$. Let $\mathbf{S}$ be a finite set of primes of $K$ including the infinite ones, and let 0 be the ring of $\mathbf{S}$-integers in $K$. Then the Schur subgroup $S(\mathfrak{o})$ of the Brauer group $B(0)$ is defined, in analogy with $S(K)$, via representations of finite groups on finitely generated projective o-modules. It is easy to see that $S(\mathfrak{o}) \subseteq$ $S(K) \cap B(\mathfrak{o})$. We shall show that there is equality in the case of $K$ a purely cyclotomic extension $\mathbb{Q}\left(\varepsilon_{m}\right)$ of $\mathbb{Q}$ (where $\varepsilon_{m}$ is an $m$ th root of 1 ).
\end{abstract}

Introduction. For any commutative ring $\mathfrak{o}$, one can define the Schur subgroup $S(\mathfrak{o})$ of the Brauer group $B(\mathfrak{o})$ as follows: Let $G$ be a finite group, and $\rho: G \rightarrow$ GL $(M)$ a homomorphism of $G$ to the automorphism group of a projective module $M$ over $\mathfrak{o}$ with the property that the o-span o $\rho G$ of $\rho G$ is an Azumaya algebra over o. In this case we denote the Brauer class $[\mathfrak{o} \rho(G)]$ by $\beta(\rho)$. Then $S(\mathfrak{o})$ consists of the Brauer classes $\beta(\rho)$ as $\rho$ runs over all such homomorphisms of all finite groups $G$. Alternatively, one can define $S(\mathfrak{o})$ to be the set of all Brauer classes $[\Lambda]$ where $\Lambda$ runs over all Azumaya algebras which are homomorphic images of some group algebra $\mathfrak{o} G$ ( $G$ finite). It is easy to see, as in the case $\mathfrak{o}=$ a field, that $S(\mathfrak{o})$ is indeed a subgroup of $B(\mathfrak{o})$ (see [D-M 1]).

Suppose now that the canonical map $B(\mathfrak{o}) \rightarrow B(K)$ is injective (this is the case if $\mathfrak{o}$ is a Dedekind domain, e.g., see [O-S]). Then it is clear that

$$
S(\mathfrak{o}) \subseteq S(K) \cap B(\mathfrak{o})
$$

and E. B. Williams has asked for conditions under which there is equality in the following situation: $K$ is a finite abelian extension of $\mathbb{Q}$, and $\mathfrak{o}$ is the ring of $\mathbf{S}$ integers in $K$ for a finite set of places $\mathbf{S}$ of $K$. We shall prove the following theorem.

THEOREM. Let $K$ be a cyclotomic field $\mathbb{Q}\left(\varepsilon_{m}\right)$ where $m$ is a positive integer, and let $\mathbf{S}$ be any finite set of primes (containing the infinite primes). Then if $\mathrm{o}$ is the ring of $S$-integers,

$$
S(\mathfrak{o})=S(K) \cap B(\mathfrak{o}) .
$$

For other results on $S(\mathfrak{o})$, see [D-M 1 and D-M 2].

Received by the editors March 19, 1987.

1980 Mathematics Subject Classification (1985 Revision). Primary 20C10, 16A26; Secondary 12A60, 16A16, 16A18, 12A35.

Key words and phrases. Schur subgroup, Brauer group, Azumaya algebras, integral representations, representations of finite groups.

Support by N.S.E.R.C. grant \#A8778 gratefully acknowledged.

(C) 1988 American Mathematical Society $0002-9939 / 88 \$ 1.00+\$ .25$ per page 
The proof. For any abelian group $A$ and any positive integer $q$, let $A_{q}$ denote the $q$-primary subgroup of $A$-that is, the subgroup of $A$ of those elements whose order divides some power of $q$.

Let $K=\mathbb{Q}\left(\varepsilon_{m}\right)$. We may assume that $m \not \equiv 2(\bmod 4)$. We must show that an arbitrary element $\alpha \in S(K) \cap B(\mathfrak{o})$ is in $S(\mathfrak{o})$. Since $B(K)$ is a torsion group, we may write $\alpha=\sum \alpha_{q}$ where $q$ runs over the rational primes and $\alpha_{q}$ is the $q$-primary component of $\alpha$. This allows us to assume that $\alpha=\alpha_{q}$, i.e. that $\alpha$ is $q$-primary. By the "root of unity theorem" of Benard (see Theorem 6.1, $[\mathbf{Y}]$ ), we can also assume that $q \mid 2 m$.

If $p$ is any rational prime, possibly $\infty$, and $L$ is an algebraic number field, then the $p$-local invariants of a Brauer class $\gamma \in B(L)$ are, by definition, the Hasse invariants (with values in $\mathbb{Q} / \mathbb{Z}$ ) of $\gamma$ at the primes of $L$ which lie above $p$-see Definition 6.4, [Y]. The Benard-Schacher theorem says that the Hasse invariants of each $\gamma$ in $S(K)$ are "uniformly distributed", i.e. for each $p$, the $p$-local invariants of $\gamma$ are determined (in a very explicit way) by any one of them (Theorem $6.1,[\mathbf{Y}]$ ). The $p$-local component of $\gamma$ is defined to be the Brauer class $\gamma(p)$ whose $p$-invariants coincide with those of $\gamma$ and whose other Hasse invariants are 0 . Thus $\gamma=\sum_{p} \gamma(p)$.

Let $M / L$ be a Galois extension with Galois group $\mathscr{G}(M / L)$. We shall denote the cohomology group $H^{2}\left(\mathscr{G}(M / L), M^{*}\right)$ by $H^{2}(M / L)$ and identify it with a subgroup of $B(L)$ in the usual way. Let $\mu(M)$ denote the group of roots of unity in $M$. There is a canonical map of $H^{2}(\mathscr{G}(M / L), \mu(M))$ into $H^{2}\left(\mathscr{G}(M / L), M^{*}\right)$ whose image will be denoted by $H_{c}^{2}(M / L)$. The Brauer-Witt theorem (Chapter $3,[\mathbf{Y}]$ ) says that $S(L)$ is the union of all $H_{c}^{2}(M / L)$ as $M$ runs over all cyclotomic extensions $M=L\left(\varepsilon_{n}\right)$ of $L$.

Suppose that $q^{r} \| m$.

LEMMA 1. The p-components $\alpha(p)$ of $\alpha$ are also in $S(K) \cap B(\mathfrak{o})$. Moreover $\alpha(p)=K \otimes \beta$ where $\beta \in H_{c}^{2}\left(\mathbb{Q}\left(\varepsilon_{p q^{r}}\right) / \mathbb{Q}\left(\varepsilon_{q^{r}}\right)\right)$ is a $p$-local $(\beta=\beta(p))$ Brauer class of $\mathbb{Q}\left(\varepsilon_{q^{r}}\right)$.

Here $K \otimes \beta$ stands for the Brauer class of $K$ obtained from $\beta$ by "restriction" (extension of scalars).

ProOF. By a theorem of Janusz $[\mathbf{J}]$, there is a $\beta \in S\left(\mathbb{Q}\left(\varepsilon_{q^{r}}\right)\right)_{q}$ such that $\alpha=K \otimes \beta$. Benard, Schacher, and Yamada have characterized $S\left(\mathbb{Q}\left(\varepsilon_{q^{r}}\right)\right)_{q}$ in terms of Hasse invariants (pp. 135-139, [Y]). Namely $S\left(\mathbb{Q}\left(\varepsilon_{q^{r}}\right)\right)_{q}$ consists of all uniformly distributed Brauer classes in $B\left(\mathbb{Q}\left(\varepsilon_{q^{r}}\right)\right)$ whose $p$-local invariants have order dividing $\left(p-1, q^{r}\right)$ for each (rational) prime $p$; there is one exception: if $q=2$ and $p \equiv-1 \bmod 2^{r}$, then the $p$-local invariant is 0 . It follows that $S\left(\mathbb{Q}\left(\varepsilon_{q^{r}}\right)\right)_{q}$ is the direct sum of its $p$-local components - the latter are the (cyclic) subgroups of $S\left(\mathbb{Q}\left(\varepsilon_{q^{r}}\right)\right)_{q}$ which are 0 locally at all primes of $\mathbb{Q}\left(\varepsilon_{q^{r}}\right)$ not above $p$. Therefore if we express $\beta$ as the sum $\sum \beta(p)$ of its $p$-local components, each component lies in $S\left(\mathbb{Q}\left(\varepsilon_{q^{r}}\right)\right)_{q}$ and furthermore we can assume it is 0 if the corresponding $p$ component $\alpha(p)$ of $\alpha$ is 0 . It is also clear that $\alpha(p)=K \otimes \beta(p)$ and, in particular that $\alpha(p) \in S(K)$. Since $B(\mathfrak{o})$ consists of the classes in $B(K)$ whose Hasse invariants are 0 outside of $\mathbf{S}$ (cf. Theorem 6.33 and Proposition 6.34, [O-S]), it follows at once that $\alpha(p) \in S(K) \cap B(\mathfrak{o})$. It follows from Lemma 8.5 and Theorem 8.6, [Y], that $\beta(p) \in H_{c}^{2}\left(\mathbb{Q}\left(\varepsilon_{p q^{r}}\right) / \mathbb{Q}\left(\varepsilon_{q^{r}}\right)\right)$. This finishes the proof of Lemma 1 .

By Lemma 1, we can now assume that $\alpha$ is $p$-local as well being $q$-primary. 
LEMMA 2. The cohomology class $\alpha$ contains a cocycle $f \in Z^{2}\left(K\left(\varepsilon_{p}\right) / K\right)$ all of whose values are in $\left\langle\varepsilon_{q^{r}}\right\rangle$.

PROOF. Because of our identifications,

$$
H_{c}^{2}\left(\mathbb{Q}\left(\varepsilon_{p q^{r}}\right) / \mathbb{Q}\left(\varepsilon_{q^{r}}\right)\right) \subseteq H_{c}^{2}\left(\mathbb{Q}\left(\varepsilon_{p^{m}}\right) / \mathbb{Q}\left(\varepsilon_{q^{r}}\right)\right) .
$$

Therefore since $\alpha=K \otimes \beta$ where $\beta$ is as in Lemma 1 , we get $\alpha \in H_{c}^{2}\left(K\left(\varepsilon_{p}\right) / K\right)$ and so Lemma 2 follows at once since $\alpha$ is $q$-primary.

LEMMA 3. If $\alpha \neq 0$, then $p$ lies below a prime of $\mathbf{S}$ and does not divide $m$.

PROOF. If $p$ were a divisor of $m$, then $K$ would contain $\varepsilon_{p}$ resp. $\varepsilon_{4}$ if $p=2$; by Proposition 4.8 and Corollary 5.4 of $[\mathbf{Y}]$, the Schur subgroup of a completion $K_{\mathfrak{p}}$ of $K$, at any prime $\mathfrak{p}$ lying over $p$, would then be trivial, so $\alpha$ also would be trivial. On the other hand, the fact that $\alpha \in B(\mathfrak{o})$ means exactly that its local components are 0 at all $\mathfrak{p}$ not in $\mathbf{S}$.

We now define $A$ be the the crossed-product algebra

$$
A=\left(K\left(\varepsilon_{p}\right) / K, f\right)=\sum_{\sigma \in \mathscr{G}} K\left(\varepsilon_{p}\right) u_{\sigma}
$$

where $\mathscr{G}=\mathscr{G}\left(K\left(\varepsilon_{p}\right) / K\right)$. Of course $A \in \alpha$. Similarly we define the order

$$
\Lambda=\left(\mathfrak{o}\left[\varepsilon_{p}\right] / \mathfrak{o}, f\right)=\sum_{\sigma \in \mathscr{S}} \mathfrak{o}\left[\varepsilon_{p}\right] u_{\sigma}
$$

Thus $A=K \otimes \Lambda$.

LEMMA 4. $\Lambda$ is an Azumaya algebra over o with Brauer class $[\Lambda]=\alpha$.

Proof. Let $\mathfrak{p}$ be any prime not in $\mathbf{S}$. Then

$$
\hat{\mathfrak{o}}_{\mathfrak{p}} \otimes \Lambda=\sum_{\sigma \in \mathscr{G}} \hat{\mathfrak{o}}_{\mathfrak{p}} \otimes \mathfrak{o}\left[\varepsilon_{p}\right] u_{\sigma}
$$

is an $\hat{\mathfrak{o}}_{\mathfrak{p}}$-order in the $K_{\mathfrak{p}}$-algebra

$$
K_{\mathfrak{p}} \otimes A=\sum_{\sigma \in \mathscr{G}} K_{\mathfrak{p}} \otimes K\left(\varepsilon_{p}\right) u_{\sigma}
$$

Let $L_{1}, \ldots, L_{g}$ be the completions of $L=K\left(\varepsilon_{p}\right)$ at the primes lying above $\mathfrak{p}$. Then the $K_{\mathfrak{p}}$-algebra $K_{\mathfrak{p}} \otimes K\left(\varepsilon_{p}\right)$ is (isomorphic to) the direct sum $L_{1} \oplus \cdots \oplus L_{g}$. We shall now show that $\hat{\mathfrak{o}}_{\mathfrak{p}} \otimes \mathfrak{o}\left[\varepsilon_{p}\right]$ is likewise the direct sum of the rings of integers $\mathfrak{D}_{i}$ of the $L_{i}$, after identifying by means of this isomorphism.

Since $\hat{\mathfrak{o}}_{\mathfrak{p}} \otimes \mathfrak{o}\left[\varepsilon_{p}\right] \subseteq \bigoplus \mathfrak{D}_{i}$ and both are $\hat{\mathfrak{o}}_{\mathfrak{p}}$-orders on a separable algebra, it suffices to show that they have the same discriminant (cf. [MO]). Now the discriminant of $\hat{\mathfrak{o}}_{\mathfrak{p}} \otimes \mathfrak{o}\left[\varepsilon_{p}\right]$ is $d\left(\mathfrak{o}\left[\varepsilon_{p}\right] / \mathfrak{o}\right) \hat{\mathfrak{o}}_{\mathfrak{p}}$ where $d\left(\mathfrak{o}\left[\varepsilon_{p}\right] / \mathfrak{o}\right)$ is the discriminant of $\mathfrak{o}\left[\varepsilon_{p}\right] / \mathfrak{o}$. The discriminant of $\bigoplus \mathfrak{D}_{i}$ is the product of the $d\left(\mathfrak{D}_{i} / \hat{\mathfrak{o}}_{\mathfrak{p}}\right)$ and thus is equal to $d\left(\mathfrak{o}\left[\varepsilon_{p}\right] / \mathfrak{o}\right) \hat{\mathfrak{o}}_{\mathfrak{p}}$ by Proposition 5 , Chapter I, [C-F].

Let $\mathscr{G}_{1} \subseteq \mathscr{G}$ be the stabilizer of the summand $L_{1}$-it is the decomposition group of the prime belonging to $L_{1}$. Consider the crossed-product order $\Lambda_{1}=\left(\mathfrak{D}_{1} / \hat{\mathfrak{o}}_{\mathfrak{p}}, f_{1}\right)$ where $f_{1}$ is the restriction of $f$ to $\mathscr{G}_{1}$. Now $f_{1}$ is split since $f$ splits at $\mathfrak{p}$. Since $\mathfrak{p}$ does not ramify in $\mathfrak{D}_{1}, \Lambda_{1}$ is a maximal order by a theorem of Auslander and Goldman-see Theorem 28.5, [C-RI]. Since $\hat{\mathfrak{o}}_{\mathfrak{p}} \otimes \mathfrak{o}\left[\varepsilon_{p}\right]$ is the direct sum of the $\mathfrak{D}_{i}$, it 
follows that $\Lambda$ is also maximal by a theorem of Merklen (Proposition 1, (iv), [M]). Since $K \otimes \Lambda=A$ and the local Hasse invariants of $A$ are all 0 at the primes of 0 (i.e. the primes $\mathfrak{p} \notin \mathbf{S}$ ), it follows that $\Lambda$ is an Azumaya algebra over $\mathfrak{o}$ and $\Lambda \in \alpha$ (cf. $[\mathrm{D}-\mathrm{I}]$ ).

Thus to prove our theorem, it suffices to prove that $\Lambda \in S(\mathfrak{o})$.

Let $G$ be the extension of $\mathscr{G}$ by $\mu(L)$, corresponding to the cocycle $f$. Then we can view $G$ as the group generated by $\mu(L)$ and the $u_{\sigma}$ in $\Lambda$. Moreover it is clear that $G$ spans $\Lambda$ over $\mathfrak{o}$, and so $\Lambda \in S(\mathfrak{o})$ as desired.

\section{REFERENCES}

[C-F] J. W. S. Cassels and A. Frohlich, Algebraic number theory, Thompson Book, Washington, D. C., 1967.

[C-RI] C. W. Curtis and I. Reiner, Methods of representation theory with applications to groups and orders, vol. I, Pure and Appl. Math., Wiley, New York, 1981.

[D-I] F. DeMeyer and E. Ingraham, Separable algebras over commutative rings, Lecture Notes in Math., vol. 181, Springer-Verlag, Berlin and New York, 1971.

[D-M 1] F. R. DeMeyer and R. A. Mollin, The Schur group of a commutative ring, J. Pure Appl. Math. 35 (1985), 117-122.

[D-M 2] _ , The Schur group, Lecture Notes in Math., vol. 1142, Springer-Verlag, Berlin and New York, pp. 205-210.

[J] G. J. Janusz, The Schur group of cyclotomic fields, J. Number Theory 7 (1975), 345-352.

[M] H. Merklen, Hereditary crossed product orders, Pacific J. Math. 74 (1978), 391-406.

[MO] I. Reiner, Maximal orders, L. M. S. Monographs 5, Academic Press, London, 1975.

[O-S] M. Orzech and C. Small, The Brauer group of commutative rings, Lecture Notes in Pure and Appl. Math., vol. 11, Marcel Dekker, New York, 1975.

[Y] T. Yamada, The Schur subgroup of the Brauer group, Lecture Notes in Math., vol. 397, Springer-Verlag, Berlin and New York, 1974.

Department of Mathematics and Statistics, MCMaster University, HamilTON, ONTARIO L8S 4K1, CANADA 